\title{
BIODISPONIBILIDADE DE CÁDMIO EM FERTILIZANTES FOSFATADOS ${ }^{(1)}$
}

\author{
Veridiana Cardoso Gonçalves ${ }^{(2)}$, Egon José Meurer ${ }^{(3)}$; Fernanda Roberta Pereira \\ Tatsch $^{(4)}$, Sheila Andréia Carvalho ${ }^{(4)} \&$ Olimpio Abadio dos Santos Neto ${ }^{(5)}$
}

\begin{abstract}
RESUMO
Os fertilizantes fosfatados apresentam em sua composição metais pesados, dentre eles o Cd, que é potencialmente tóxico ao homem, animais e plantas. $\mathrm{O}$ objetivo deste trabalho foi verificar a biodisponibilidade do $\mathrm{Cd}$ contido em fertilizantes fosfatados. Seis fertilizantes fosfatados (dois fosfatos naturais, dois superfosfatos simples, um superfosfato triplo e um fosfato monoamoniado) foram selecionados para o estudo de biodisponibilidade em experimento realizado em casa de vegetação, utilizando-se Avena strigosa como indicadora da biodisponibilidade do $\mathrm{Cd}$. $\mathrm{O}$ Cd presente nos fertilizantes fosfatados mostrou-se biodisponível, pois foi encontrado nas raízes e na parte aérea da aveia preta. Os fertilizantes fosfatados com maiores teores de $\mathrm{Cd}$ aumentaram a quantidade desse metal acumulado nas plantas
\end{abstract}

Termos de indexação: elemento-traço, fosfatos comerciais, aveia, metal pesado.

\section{SUMMARY: CADMIUM BIOAVAILABILITY IN PHOSPHATE FERTILIZERS}

Phosphate fertilizers contain heavy metals, including $C d$, which is potentially toxic to humans, animals and plants. The objective of this study was to assess the bioavailability of Cd contained in phosphate fertilizers. Six phosphate fertilizers ( $t$ wo natural phosphates, two simple superphosphate, a triple superphosphate and a monoammonia phosphate) were selected for the study of bioavailability in a greenhouse experiment, using Avena strigosa as an indicator

(1) Parte da Dissertação de Mestrado do primeiro autor. Trabalho apresentado no XXXI Congresso Brasileiro de Ciência do Solo, 2007 (Gramado, RS).

(2) Pós-graduando em Ciência do Solo, Faculdade de Agronomia, Universidade Federal do Rio Grande do Sul (UFRGS). Porto Alegre/RS. E-mail: vericg79@yahoo.com.br

(3) Professor Associado, Departamento de Solos (UFRGS). Bolsista do CNPq. E-mail: egon.meurer@ufrgs.br

(4) Bolsista de Iniciação Científica, Departamento de Solos, UFGRS. Email: agronomia81@gmail.com e sheila_carv@yahoo.com.br

(5) Engenheiro-Agrônomo, Agência Goiana de Defesa Agropecuária (Agrodefesa). Av. Portugal, no 591, Setor Oeste. Goiania-GO. CEP 74140-020. E-mail: olimpioneto10@hotmail.com 
of the bioavailability of $C d$. The $C d$ was bioavailable in phosphate fertilizers, since it was found in roots and shoots of the oat. The phosphate fertilizers with higher Cd levels increased its accumulation in plants.

Index terms: trace-element, commercials phosphates, Avena strigosa, heavy metal.

\section{INTRODUÇÃO}

O Pé o macronutriente que freqüentemente limita a produção e a produtividade das culturas em muitos solos brasileiros. As principais fontes de $\mathrm{P}$ usadas na agricultura para suprir a necessidade deste nutriente são os fosfatos naturais, os parcialmente acidulados e os solúveis. A matéria-prima para a obtenção destes produtos são as rochas fosfáticas que apresentam em sua composição, além do $\mathrm{P}$, diversas impurezas, dentre elas o Cd, em variada concentração (McLaughin \& Singh, 1999; Prochnow et al., 2001).

Adubações fosfatadas sucessivas podem incrementar as quantidades de $\mathrm{Cd}$ em solos e plantas (Gimeno-Garcia et al., 1996; Marchiori Jr., 2003), o que pode resultar em impactos no ambiente pela contaminação de águas superficiais e subsuperficiais, possibilitando a entrada deste metal na cadeia alimentar. $\mathrm{O}$ Cd é prejudicial à saúde humana e animal (McLaughin \& Singh, 1999; Uemura 2000; Waalkes, 2000) e pode interferir no crescimento e desenvolvimento das plantas mesmo em baixas concentrações (McLaughin \& Singh, 1999; Matsuda et al., 2000).

O objetivo deste trabalho foi avaliar a biodisponibilidade do Cd em fertilizantes fosfatados pelo seu acúmulo em plantas de aveia preta.

\section{MATERIAL E MÉTODOS}

O experimento foi realizado em casa de vegetação com amostra de um Nitossolo Vermelho distroférrico latossólico (NVdf), coletado no Estado do Rio Grande do Sul, na profundidade de 0-0,20m. Após a coleta, o solo foi corrigido para $\mathrm{pH}$ 5,5 pela incubação, durante 30 dias, com carbonato de cálcio $\left(\mathrm{CaCO}_{3}\right)$ e carbonato de magnésio $\left(\mathrm{MgCO}_{3}\right)$, na relação molar 3:1. Os tratamentos consistiram em duas doses de P, 250 e $500 \mathrm{mg} \mathrm{kg}^{-1}$ de $\mathrm{P}_{2} \mathrm{O}_{5}$ no solo, utilizando-se como fonte de $\mathrm{P}$ amostras de seis fertilizantes fosfatados comercializados no Brasil (Quadro 1) selecionadas em estudo anterior (Bizarro, 2007, 2008). O experimento foi instalado em delineamento completamente casualizado com três repetições por tratamento. $\mathrm{O} \mathrm{N}$ e o $\mathrm{K}$ foram supridos na forma de soluções de $\mathrm{NH}_{4} \mathrm{NO}_{3}$ e $\mathrm{KCl}$ em doses de 40 e $25 \mathrm{mg} \mathrm{kg}^{-1}$ de solo. Para suprir o nitrogênio nos tratamentos em que foi utilizado fosfato monoamoniado como fonte de $\mathrm{P}_{2} \mathrm{O}_{5}$, considerou- se o teor de $\mathrm{N}$ do fertilizante, completando-se a dose com $\mathrm{NH}_{4} \mathrm{NO}_{3}$.

As unidades experimentais foram vasos plásticos com capacidade para $4 \mathrm{~kg}$ de solo. Após a correção do $\mathrm{pH}$ e adubação, foram colocadas para germinar cinco sementes de aveia preta (Avena strigosa) por vaso, que foi colhida aos 60 dias da semeadura. Após a colheita, as plantas foram separadas em parte aérea e raízes e secas em estufa com circulação forçada de ar a $65^{\circ} \mathrm{C}$. Este material foi moído e a extração do $\mathrm{Cd}$ no tecido foi realizada pelo método nitroperclórico, (Tedesco et al., 1995), modificado por Scolmeister (1999). O solo dos vasos após a coleta das plantas foi seco, passado em peneira com malha de $2 \mathrm{~mm}$ e analisado quanto ao teor de $\mathrm{Cd}$ pelo método Usepa 3050B, segundo USEPA (1998). A quantificação dos teores de Cd nas amostras de plantas e solo foi realizada por espectrometria de emissão atômica por plasma acoplado indutivamente (ICP - AES). A análise estatística foi realizada pelo programa SANEST (Zonta et al., 1984) e a comparação entre as médias pelo teste de Tukey a $5 \%$.

\section{RESULTADOS E DISCUSSÃO}

Nas raízes e parte aérea das plantas houve, acúmulo de Cd resultante da aplicação dos fertilizantes fosfatados ao Nitossolo (Figuras 1 e 2). Nas raízes da aveia, o maior acúmulo do metal ocorreu com a aplicação da dose equivalente a $500 \mathrm{mg} \mathrm{kg}^{-1}$ de $\mathrm{P}_{2} \mathrm{O}_{5}$ no solo do fosfato monoamoniado MAP3 $(2,5 \mu \mathrm{g}$ planta de $\mathrm{Cd}$ ) no qual a concentração de $\mathrm{Cd}$ atingiu 31,05 mg $\mathrm{kg}^{-1}$ de Cd (Quadro 1), mas não alterou estatisticamente as quantidades de $\mathrm{Cd}$ acumulado nas raízes com a aplicação dos fosfatos naturais FN3 e FN4 e do superfosfato simples SS2. Vale mencionar que o SS2, embora com um teor de Cd de $12,34 \mathrm{mg} \mathrm{kg}^{-1}$, inferior aos teores do FN3 e FN4 (42,60 e 30,91 $\mathrm{mg} \mathrm{kg}^{-1}$ de Cd, respectivamente), acumulou teores similares de $\mathrm{Cd}$ nas raízes das planta. O P do superfosfato simples é considerado como prontamente disponível às plantas, desde que ele seja, na quase totalidade, "solúvel em água" em contraposição àquele contido nos fosfatos naturais. Estes resultados indicam que somente o teor total de Cd contido no fertilizante e o seu grau de solubilidade "em água" não são características determinantes da biodisponibilidade do $\mathrm{Cd}$ para as plantas que crescem em solos fertilizados com estes materiais. 
Quadro 1. Fertilizantes fosfatados, teor de $\mathrm{Cd}, \mathrm{P}_{2} \mathrm{O}_{5}$ total e quantidades de $\mathrm{Cd}$ adicionadas à amostra do Nitossolo Vermelho distroférrico latossólico

\begin{tabular}{|c|c|c|c|c|c|}
\hline Tratamento & Fertilizante & Sigla & $\begin{array}{l}\text { Teor de } \\
\text { Cd }^{1}\end{array}$ & $\begin{array}{l}\mathrm{P}_{2} \mathrm{O}_{5} \text { total } \\
\text { adicionado ao solo }\end{array}$ & $\begin{array}{c}\text { Cd } \\
\text { adicionado ao solo }\end{array}$ \\
\hline & & & $\mathrm{mg} \mathrm{kg-1}$ & $\mathrm{mg} \mathrm{kg}-1$ & $\mu \mathrm{g} \mathrm{kg}^{-1}$ \\
\hline $\mathrm{T} 1$ & $\mathrm{NH}_{4} \mathrm{H}_{2} \mathrm{PO}_{4}$ & Test & \multirow{2}{*}{0,00} & 250 & 0,00 \\
\hline $\mathrm{T} 2$ & $\mathrm{NH}_{4} \mathrm{H}_{2} \mathrm{PO}_{4}$ & Test & & 500 & 0,00 \\
\hline $\mathrm{T} 3$ & Fosfato Natural & FN3 & \multirow{2}{*}{42,60} & 250 & 10,65 \\
\hline $\mathrm{T} 4$ & Fosfato Natural & FN3 & & 500 & 21,30 \\
\hline $\mathrm{T} 5$ & Fosfato Natural & FN4 & \multirow{2}{*}{30,91} & 250 & 7,73 \\
\hline $\mathrm{T} 6$ & Fosfato Natural & FN4 & & 500 & 15,45 \\
\hline $\mathrm{T} 7$ & Superfosfato Simples & $\mathrm{SS} 2$ & \multirow{2}{*}{12,34} & 250 & 3,08 \\
\hline $\mathrm{T} 8$ & Superfosfato Simples & SS2 & & 500 & 6,15 \\
\hline $\mathrm{T} 9$ & Superfosfato Simples & SS3 & \multirow{2}{*}{1,49} & 250 & 0,37 \\
\hline $\mathrm{T} 10$ & Superfosfato Simples & SS3 & & 500 & 0,75 \\
\hline $\mathrm{T} 11$ & Superfosfato Triplo & $\mathrm{ST} 2$ & \multirow{2}{*}{25,92} & 250 & 6,48 \\
\hline $\mathrm{T} 12$ & Superfosfato Triplo & ST2 & & 500 & 12,95 \\
\hline $\mathrm{T} 13$ & Fosfato monoamoniado & MAP3 & \multirow{2}{*}{31,05} & 250 & 7,75 \\
\hline $\mathrm{T} 14$ & Fosfato monoamoniado & MAP3 & & 500 & 15,50 \\
\hline
\end{tabular}

${ }^{1}$ Extração com ácido nitroperclórico

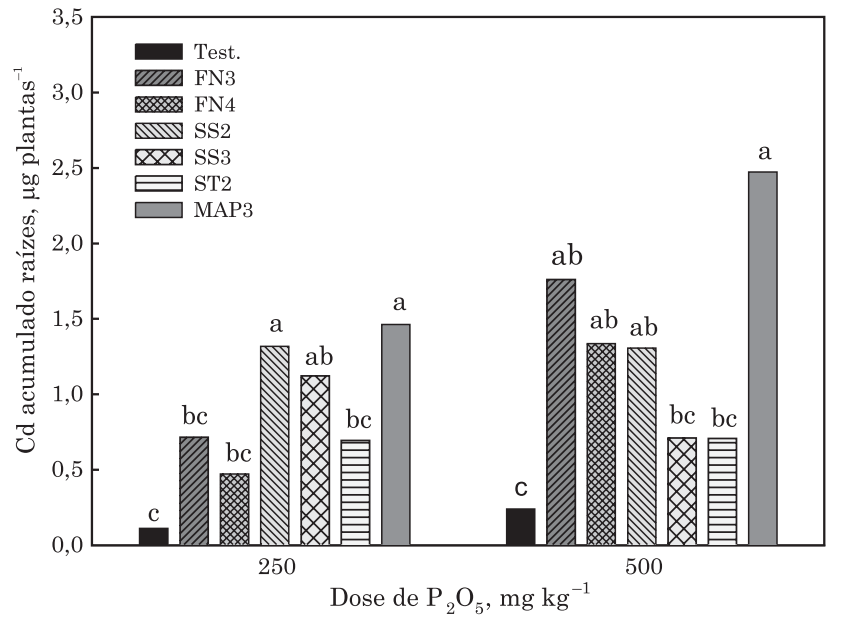

Figura 1. Quantidade de Cd acumulado nas raízes da aveia preta pela adição de fertilizantes fosfatados a um Nitossolo Vermelho distroférrico latossólico. Médias seguidas por mesma letra na dose não diferem pelo teste de Tukey a $5 \%$.

A quantidade de $\mathrm{Cd}$ acumulado na parte aérea das plantas, para a dose de $250 \mathrm{mg} \mathrm{kg}^{-1} \mathrm{de}$ $\mathrm{P}_{2} \mathrm{O}_{5}$ no solo, com exceção do ST2 e MAP3, foi menor do que a acumulada nas raízes. Destacase que, para as fontes FN4 e SS3, as quantidades de $\mathrm{Cd}$ acumulado na parte aérea da aveia não

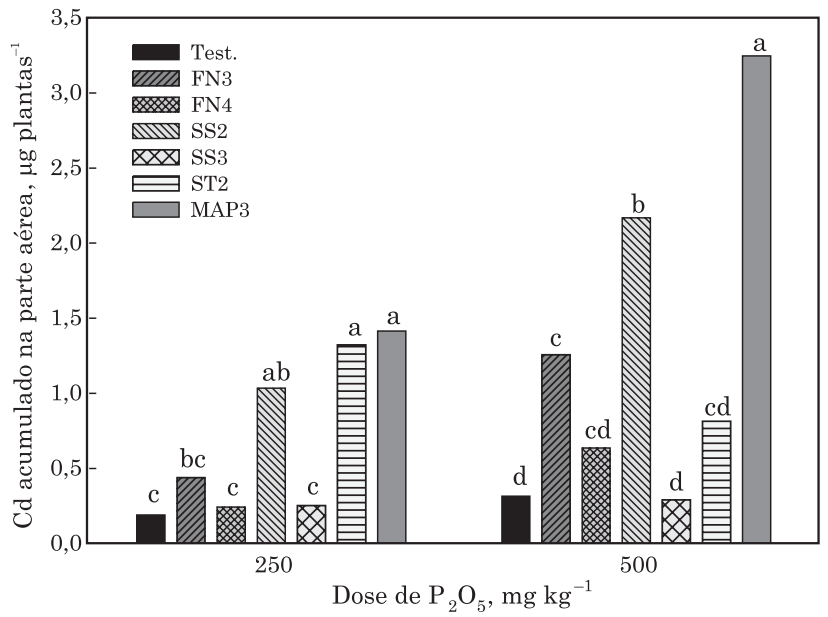

Figura 2. Quantidade de Cd acumulado na parte aérea da aveia preta pela adição de fertilizantes fosfatados a um Nitossolo Vermelho distroférrico latossólico. Médias seguidas por mesma letra na dose não diferem pelo teste de Tukey a $5 \%$.

diferiram da testemunha. Quando se aplicaram $500 \mathrm{mg} \mathrm{kg}^{-1}$ de $\mathrm{P}_{2} \mathrm{O}_{5}$ no solo, as quantidades de $\mathrm{Cd}$ acumulado na parte aérea da aveia para as fontes MAP3 e SS2 foram significativamente maiores do que as acumuladas para as demais fontes (Figura 2). 
Estes resultados indicam que o $\mathrm{Cd}$ nas fontes de fertilizantes fosfatados utilizados são biodisponíveis, pois foi acumulado nas raízes e na parte aérea das plantas. Indicam ainda, que houve translocação do metal na planta e que a biodisponibilidade do Cd entre fontes é diferenciada.

Os resultados obtidos estão de acordo com os valores encontrados por Trevizam (2005) na parte aérea de arroz cultivado em um Latossolo VermelhoAmarelo e em um Neossolo Quartizarênico, com adição de diferentes fertilizantes fosfatados; por Oliveira Jr. (2001), na folha Y de arroz cultivado em um Latossolo Roxo e um Latossolo VermelhoAmarelo distrófico; por Oliveira (2003), em arroz cultivado em dois Latossolos, e por Mulla et al. (1980). Os autores citados mencionam em seus trabalhos teores de 0,2 a $1,7 \mathrm{mg} \mathrm{kg}^{-1}$ de Cd no tecido das plantas. Por outro lado, os valores observados discordam dos teores encontrados no tecido de milho e soja por Mortvedt (1987). Esse autor encontrou teores de Cd nas culturas entre 0,04 e 0,09 $\mathrm{mg} \mathrm{kg}^{-1}$, após longo período de adubação fosfatada com superfosfato triplo.

Embora os teores de Cd encontrados na parte aérea e raízes da aveia preta tenham sido similares, o teor nas raízes foi maior, concordando com os resultados observados por Maclean (1976) em partes vegetativas e raízes de 10 espécies de plantas.

Nos tratamentos em que foram aplicados $500 \mathrm{mg}$ $\mathrm{kg}^{-1}$ de $\mathrm{P}_{2} \mathrm{O}_{5}$, a quantidade de Cd encontrado na parte aérea não atingiu valores considerados tóxicos. Segundo Pais \& Jones (1997), o Cd passa a ser tóxico quando seu acumulo no tecido das plantas é superior a $35 \mathrm{mg} \mathrm{kg}^{-1}$. O incremento de Cd no tecido das plantas com a adição de fertilizantes fosfatados também foi observado por outros autores: Oliveira Júnior (2001), em arroz, por Gonçalves Jr. \& Pessoa (2002) e Gonçalves Jr. et al. (2000), na cultura da soja.

Quanto ao teor total de Cd no solo (dados não apresentados), os valores encontrados foram inferiores a $0,2 \mathrm{mg} \mathrm{kg}^{-1}$ (valor abaixo do limite de detecção do espectrômetro de emissão atômica por plasma acoplado indutivamente (ICP-AES)). A adição ao solo das doses de 250 e $500 \mathrm{mg} \mathrm{kg}^{-1} \mathrm{de}$ $\mathrm{P}_{2} \mathrm{O}_{5}$ em fontes com diferentes teores de $\mathrm{Cd}$ não foi suficiente para detectar o incremento do teor deste metal pesado no solo. Os teores totais de $\mathrm{Cd}$ no solo encontrados neste trabalho estão abaixo dos considerados como críticos para solos agrícolas (10 mg kg-1), segundo Alloway (1995) e CETESB (2001). Em experimentos de longa duração utilizando superfosfatos para as plantas, Mulla et al. (1980) e Mortvedt (1987) também não encontraram incrementos significativos no teor de Cd no solo.

\section{CONCLUSÕES}

1. O Cd nos fertilizantes fosfatados foi biodisponível, pois, embora não detectado no solo, foi encontrado nas raízes e parte aérea da aveia preta.

2. A biodisponibilidade do $\mathrm{Cd}$ entre as fontes testadas não foi a mesma, pois as quantidades acumuladas nas raízes e translocadas para a parte aérea das plantas foram diferentes.

\section{LITERATURA CITADA}

ALLOWAY, B.J. Heavy metals in soils. 2.ed. Glasgow, Blackie Academic, 1995. 364p.

BIZARRO, V.G. Teor e biodisponibilidade de cádmio em fertilizantes fosfatados. Porto Alegre, Universidade Federal do Rio Grande do Sul, 2007. 65p. (Tese de Mestrado)

BIZARRO, V.G.; MEURER E.J. \& TATSCH, F.R.P. Teor de cádmio em fertilizantes fosfatados. Ci. Rural, 38:247-250, 2008 .

COMPANHIA DE TECNOLOGIA DE SANEAMENTO AMBIENTAL - CETESB. Relatório de estabelecimento de valores orientadores para solos e águas subterrâneas no Estado de São Paulo. São Paulo, 2001. 247p.

GIMENO-GARCIA, E.; ABREU, V. \& BOLUDA, R. Heavy metals incidence in the application of inorganic fertilisers and pesticide to rice farming soils. Environ. Poll., 92:19$25,1996$.

GONÇAVES JR., A.C.; LUCHESE, E.B. \& LENZI, E. Avaliação da fitodisponibilidade de cádmio, chumbo e cromio, em Latossolo Vermelho-Escuro tratado com fertilizantes comerciais. Química Nova, 23:173-177, 2000.

GONÇALVES JR., A.C. \& PESSOA, A.C.S. Fitodisponibilidade de cádmio, chumbo e cromio, em soja cultivada em Argissolo Vermelho eutrófico a partir de adubos comerciais. Sci. Agr., 3:19-23, 2002.

MACLEAN, A.J. Cadmium in different plant species and its availiability in soils as influenced by organic matter and additions of lime, P, Cd and Zn. Canadian J. Soil Sci., 56:129-138, 1976

MARCHIORI JR., M. Levantamento inicial de chumbo, cádmio, níquel, cromo e cobre em pomares de produção comercial no Estado de São Paulo. Jaboticabal, Universidade Estadual Paulista, Jaboticabal, 2003. 138p. (Tese de Doutorado)

MATSUDA, A.; SOUZA, F.M. \& OSWALDO, J. Tolerância de rizóbios de diferentes procedências de zinco, cobre e cádmio. Pesq. Agropec. Bras., 37:343-355, 2000.

McLAUGHLIN, M.J. \& SINGH, B.R. Cadmium in soil and plants. Dordrecht, Kluwer, 1999. 269p.

MORTVEDT, J.J. Cadmium levels in soils and plants from long-term soil fertility experiments in the United States of America. J. Environ. Quality, 6:137-142, 1987. 
MUllA, D.J.; PAGE, A.L. \& ANJE, T.J.G. Cadmium accumulation on bioavailability in soils from long term phosphorus fertilization. J. Environ. Quality, 9:408-412, 1980 .

OLIVEIRA JÚNIOR, J.A. Adubos fosfatados como fonte de metais pesados - Efeito na composição do solo e do arroz. Piracicaba, Centro de Energia Nuclear na Agricultura, 2001. 81p. (Tese de Doutorado)

OLIVEIRA, S.C. Solubilidade e disponibilidade de micronutrients e metais pesados tóxicos em fertilizantes comercializados no Brasil. Piracicaba, Escola Superior de Agricultura Luíz Queiroz 2003. 156p. (Tese de Mestrado)

PAIS, I. \& JONES Jr., J.B. The handbook of trace elements. Boca Raton, St. Lucie Press, 1997. 223p.

PROCHNOW, L.I.; PLESE, L.M. \& ABREU, M.F. Bioavailibity of cadmium contained in single superphosphates produced from different Brazilian raw materials. Comm. Soil Sci. Plant Anal., 32:283-294, 2001.

SCOLMEISTER, D. Biodisponibilidade de metais pesados em solos do Rio Grande do Sul. Porto Alegre, Universidade Federal do Rio Grande do Sul, 1999. 78p. (Tese de Mestrado)
TEDESCO, M.S.; GIANELLO, C.; BISSANI, C.A.; BOHNEN, H. \& VOLKWEISS, S.V. Análises do solo, plantas e outros materiais. Porto Alegre, Universidade Federal do Rio Grande do Sul, 1995. 174p. (Boletim Técnico, 5)

TREVIZAM, A.R. Solubilidade e disponibilidade de micronutrients e elementos contaminantes em fertilizantes. Piracicaba, Centro de Energia Nuclear na Agricultura, 2005. 132p. (Tese de Mestrado)

UEMURA, T. Experimental reproduction of "Itai-itai" disease: A chronic cadmium poisoning of humanus in rats and monkeys. J. Veterinária Res., 48:15-28, 2000.

UNITED STATES ENVIRONMENTAL PROTECTION AGENCY - USEPA. Method 3050B. 1998. Disponível: $<$ http://www.epa.gov/SW-846/pdfs/3050b.pdf> Acesso em: 10 de outubro de 2005.

WAALKES, M.P. Cadmium carcinogenesis in review. J. Inorg. Biochem., 79:241-244, 2000.

ZONTA, E.P.; MACHADO, A.D. \& SILVEIRA JÚNIOR, P. Sanest: Sistema de análise estatística para microcomputadores. Registrado na secretaria especial de informática sob número 066060 - categoria A. Pelotas, Universidade Federal de Pelotas, 1984. 
